\title{
Variedades de Coffea arabica L. con manejo orgánico en Oaxaca
}

\author{
Varieties of Coffea arabica L. with organic management in Oaxaca
}

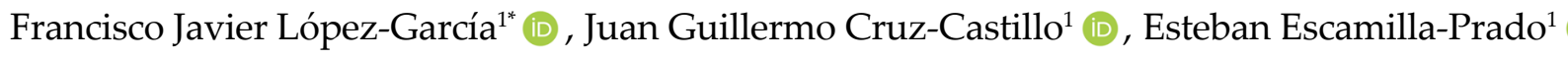 \\ ${ }^{1}$ Centro Regional Universitario Oriente, Universidad Autónoma Chapingo, km 6 Carretera Huatusco-Xalapa, 94100, Huatusco, Veracruz, México.
}

*Autor para correspondencia: logafja@hotmail.com

\section{Fecha de recepción:}

10 de enero de 2020

Fecha de aceptación:

4 de marzo de 2021

Disponible en línea:

11 de agosto de 2021

Este es un artículo en acceso abierto que se distribuye de acuerdo a los términos de la licencia Creative Commons.

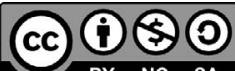

Reconocimiento-

NoComercia-

CompartirIgual 4.0

Internacional

\section{RESUMEN}

México es un importante productor de café orgánico, cosechado principalmente por productores con pequeñas parcelas sombreadas donde se utiliza una diversidad de variedades. Se evaluó la producción de fruta, el rendimiento industrial cereza-pergamino, y la calidad sensorial de la bebida de 20 variedades de café (10 de porte alto y 10 de porte bajo), durante seis ciclos de producción en Oaxaca, México. En el manejo agronómico de estos cafetos se consideraron normas de cultivo orgánico. Las variedades con mayor producción promedio de fruto en seis años fueron tres de porte bajo: Colombia Brote Café (30.2 kg/planta), Oro Azteca (29.0 kg/planta) y Catuai Amarillo (27 kg/planta). Estas variedades tuvieron un grano de tamaño grande adecuado para una preparación estilo europeo. La variedad Oro Azteca, presentó buenos atributos en aroma, acidez y cuerpo. Las variedades de porte alto con mayor rendimiento intermedio fueron: Batie, Dessie, Pluma Hidalgo 177 y Typica 947, con alrededor de 24 kg/planta. Pacamara y Blue Mountain tuvieron baja producción de fruto. Algunas variedades alcanzaron un buen sabor en taza sin importar su porte. Las anormalidades en los granos y los rendimientos agroindustriales fueron similares para todas las variedades.

\section{palabras Clave}

Café biológico, café ecológico, producción bajo sombra, evaluación sensorial del café, calidad en taza.

\section{ABStract}

Mexico is an important producer of organic coffee, mainly harvested by producers with small shade plots who use a diversity of varieties. Considering organic cultivation standards, we evaluated fruit production, cherry-parchment industrial yield, and the sensory cup quality of 20 coffee varieties (10 tall stature and 10 compact stature) during six production cycles in Oaxaca, Mexico. The varieties with the highest average fruit production in six years were three compact stature: Colombia Brote Café (30.2 kg / plant), Oro Azteca (29.0 kg / plant), and Yellow Catuai (27 kg / plant). These varieties produce large beans suitable for a European-style preparation. The Aztec Gold varieties presented good aroma, acidity and body attributes. Tall stature varieties with greater intermediate yield were Batie, Dessie, Pluma Hidalgo 177, and Typica 947, with around $24 \mathrm{~kg}$ / plant. Pacamara and Blue Mountain had low fruit production. Some varieties achieved a good taste irrespectively of their stature. Abnormalities in beans and agroindustrial yields were similar for all varieties.

\section{KEYWORDS}

Biological coffee, ecological coffee, shade coffee production, sensorial evaluation of coffee, coffee cup quality. 


\section{INTRODUCCIÓN}

El estado de Oaxaca es el cuarto productor de café (Coffea arabica L.) en México, con más de 100 mil productores, principalmente minifundistas indígenas, que cultivan $128 \mathrm{mil}$ ha y producen $200 \mathrm{mil}$ quintales de café oro (SIAP 2017). Los productores venden mayormente café pergamino, después del beneficiado húmedo del fruto fresco o cereza (Sosa et al. 2004).

Esta entidad federativa es pionera en la producción de café orgánico y tiene más de 15 mil productores que presentan rendimientos bajos (Morales 2013). En algunas regiones del estado, los productores de café generalmente presentan una diversidad productiva que amortigua efectos de fenómenos ambientales adversos y dependencia excesiva con el mercado del café. La mayoría de los cafetales son bajo sombra y pueden presentar entre 43 y 100 años de antigüedad (Cruz y Torres 2015).

La producción de café orgánico es económicamente viable y sostenible con sus limitaciones (Valkila 2009); por ejemplo, mucha fuerza de trabajo debe ser aplicada para cubrir los requerimientos nutricionales de los cafetos (Van der Vossen 2005), y bajo un contexto de emigración de productores, se limita su sostenibilidad en Oaxaca (Lewis y Runsten 2008; Morales 2013).

Existen factores ambientales que influyen en la producción y calidad del café, como la altitud de la plantación y la sombra, pero la variedad y el manejo agronómico son importantes en los sistemas de producción orgánica de café. Así, en la cadena productiva del café orgánico, la calidad del grano influida por la variedad es un requisito para aumentar las ganancias de los pequeños productores (Díaz et al. 2009). La variedad afecta significativamente la cantidad de los frutos que se cosecharán y los atributos químicos que determinan un buen café en la taza (Echeverría-Beirute et al. 2018).

El mejoramiento genético de $C$. arabica ha sido mayormente enfocado hacia el control de enfermedades y para elevar rendimientos, y no para la producción de café orgánico o con bajos insumos (Van der Vossen et al. 2015). En México, existen estudios sobre las características productivas (López-García et al. 2016; López-García y Cruz-Castillo 2019), la calidad física del grano y en taza (Escamilla et al.
2015; Partida-Sedas et al. 2019), y las propiedades nutraceúticas (Lazcano-Sánchez et al. 2015) de diversas variedades de café. Sin embargo, existe poca información sobre la producción, calidad física y en taza de los granos cosechados de variedades de $C$. arabica con manejo orgánico durante varios ciclos de producción. Así, estudios para recomendar variedades de café bajo manejo orgánico para zonas cafetaleras en México son relevantes. Particularmente, la evaluación agronómica de variedades de café con manejo orgánico en Oaxaca, durante varios ciclos productivos, no fueron encontrados en la literatura.

Los objetivos del presente estudio - utilizando 20 variedades de café bajo un manejo orgánico en Oaxaca - fueron: 1) determinar el rendimiento de café cereza durante seis años subsecuentes, y 2) evaluar la calidad física del grano, el rendimiento agroindustrial y la calidad en taza durante dos años sucesivos.

\section{Materiales y Métodos}

Se establecieron 20 variedades de café (10 de porte alto y 10 de porte bajo) (Cuadro 1), con edad de 12 meses en el Municipio de Valle Nacional, Oaxaca (17 $50^{\prime} 59.7^{\prime \prime} \mathrm{N}, 96^{\circ} 20^{\prime} 00.3^{\prime \prime} \mathrm{O}, 1,004 \mathrm{msnm}$ ). El clima fue semicálido húmedo y el suelo correspondió a una Rendzina. A 0-40 cms, el suelo tuvo un $\mathrm{pH}$ de 4.6, N total de 0.22 por ciento, $\mathrm{P}$ disponible de $12.5 \mathrm{ppm}, \mathrm{K}$ de 65 ppm, Ca de 811.0 ppm, Mg de 114 ppm, Mn de 5.6 ppm, Fe de 8.0 ppm, Cu de 0.28 ppm, Zn de 5.28 ppm, materia orgánica de 4.2 por ciento y conductividad eléctrica de $0.1741 \mu \mathrm{S}$. El muestreo y análisis del suelo se llevó a cabo de acuerdo con la norma NOM021-RECNAT-2000 (SEMARNAT 2002).

La distancia de plantación para las variedades de porte bajo fue de $1.5 \times 2.0 \mathrm{~m}$, y para las variedades de porte alto de $2.0 \times 2.5 \mathrm{~m}$, entre plantas y entre filas, respectivamente. Los tratamientos se distribuyeron conforme a un diseño experimental de bloques completos al azar, con seis repeticiones y una unidad experimental de seis plantas.

La sombra de las parcelas se estableció con árboles del género Inga, 30 días después de la plantación de los cafetos, en arreglo de marco rectangular de $8 \times 6$ $\mathrm{m}$. La parcela experimental se manejó de acuerdo con las normas de producción orgánica (CERTIMEX 2014; Sosa et al. 2004). 
Cuadro 1. Variedades de Coffea arabica de portes bajo y alto evaluadas en Valle Nacional, Oaxaca, México.

\begin{tabular}{ll}
\hline Porte bajo & Porte alto \\
\hline 1. Caturra Rojo & 11. Typica 947 \\
2. Catuai Amarillo & 12. Bourbón Salvadoreño \\
3. Caturra Amarillo & 13. Batie \\
4. Pacamara & 14. Blue Mountain \\
5. Garnica F & 15. Typica Xhantocarpa \\
6. Garnica Iquimite & 16. Pluma Hidalgo 177 \\
7. Colombia Brote Verde $(\mathrm{BV})^{*}$ & 17. Dessie \\
8. Colombia Brote Café $(\mathrm{BC})^{\dagger}$ & 18. Garnica (TCPA) \\
9. Garnica $(\mathrm{TCPB})^{\S}$ & 19. Typica Tres Cruces $(\mathrm{TC})^{\S}$ \\
10. Oro Azteca & 20. Bourbón Tres Cruces $(\mathrm{TC})^{\S}$ \\
\hline
\end{tabular}

*Brote terminal con hojas verdes; †Brote terminal con hojas cafés; §Con tres ramas plagiotrópicas por nudo, en lugar de dos.

Composta y lombricomposta de café fueron aplicadas $5 \mathrm{~T} / \mathrm{ha}(2.5 \mathrm{Kg} /$ planta) después de la cosecha. Los primeros dos años fue composta y los siguientes dos, lombricomposta. La preparación de la pulpa de café para composta y lombricomposta se hicieron de acuerdo con Sánchez et al. (1999) y Orozco et al. (1996), respectivamente. Las características físico-químicas de ambos abonos orgánicos pueden consultarse en Sanchez et al. (1999), Kasongo et al. (2011) y Orozco et al. (1996). El control de hierbas se hizo con machete, y los cafetos nunca fueron podados.

\section{Producción de café cereza}

En las 20 variedades de cafeto se determinó el peso fresco de la fruta o producción de café cereza $(\mathrm{kg}$. $\mathrm{pl}^{-1}$ ). Cada cosecha fue evaluada durante seis años de producción. La cosecha se llevó a cabo entre diciembre y febrero en cada uno de los seis periodos de producción. El origen de las variedades estudiadas puede ser consultado en Escamilla-Prado (2016), las características distintivas de estos materiales pueden observarse de forma virtual (https://www.inforural. com.mx/cafe-variedades/) y en Escamilla-Prado (2016).

\section{Calidad física del grano (rendimiento industrial, anormalidades y tamaño degrano para preparaciones)}

La calidad física del grano se determinó promediando dos ciclos productivos. El porcentaje de frutos vanos fue tomado en una muestra al azar de 500 frutos maduros por variedad, la cual se depositó en una cubeta con agua y los frutos se sumergieron por $2 \mathrm{~min}$, para separar los granos flotantes, los cuales se contabilizaron para obtener el porcentaje de frutos vanos (Rosas et al. 2008).

Para cada variedad, se seleccionaron al azar $6 \mathrm{~kg}$ de fruto, para ser despulpados con una despulpadora de discos manual (100MEX®, Ciudad de México, México). Luego se procedió a la fermentación natural, la cual tuvo una temperatura de $16{ }^{\circ} \mathrm{C}$ y un tiempo de $30 \mathrm{~h}$. Los granos se pasaron en zarandas de madera. Posteriormente, el secado se hizo por exposición al Sol hasta una humedad máxima de 12 por ciento determinada con un higrómetro (Delmhorst $G 7{ }^{\circledR}$, Arizona, Estados Unidos) (Espinosa-Solares et al. 2005). El rendimiento industrial de la conversión de café cereza a café pergamino (RCP), que define el total de café cereza por variedad para obtener un quintal, se obtuvo con la fórmula:

$\mathrm{RCP}=(57.5 \mathrm{~kg} /$ masa del café pergamino $) /$ masa del café cereza.

En esta ecuación, la cantidad $57.5 \mathrm{~kg}$ de café pergamino equivale a un quintal (1 Qq) (Espinosa-Solares et al 2005).

Para determinar el rendimiento de café pergamino a café oro (RPO), de cada variedad se tomaron $250 \mathrm{~g}$, los cuales se beneficiaron a café oro; se pesó el café oro resultante y se calculó el rendimiento con la ecuación de Espinosa-Solares et al. (2005): 
$\mathrm{RPO}=(250 \mathrm{~g} /$ masa de café pergamino $) /$ masa de café en oro

En donde, $46 \mathrm{~kg}$ de oro es el estándar nacional para un quintal de café oro (Espinosa-Solares et al. 2005).

Del café oro se analizaron muestras de $100 \mathrm{~g}$, en las que se cuantificaron los granos tipo planchuela (normal), caracol, triángulo, concha y gigante (López-García et al. 2016). Los valores de estas variables se expresaron en porcentaje.

Para medir el tamaño del grano, se usaron muestras de $100 \mathrm{~g}$ de café oro, las cuales se pasaron por las zarandas de los números 18, 17, 16, y 15 (7.44, 6.75, 6.35, $5.95 \mathrm{~mm}$ ). Posteriormente, se sumaron los porcentajes de los granos capturados en las zarandas 18 y 17, que son los que clasificaron como preparación Europea; lo mismo se hizo con los granos de las zarandas 16 y 15 , los cuales se clasificaron para la preparación Americana (López-García et al. 2016).

\section{Calidad sensorial de la bebida}

Se evaluaron los atributos aroma, acidez, cuerpo y sabor para cada variedad (Valencia et al. 2015). Un panel de cinco catadores fue seleccionados, y la escala que se utilizó varió del 1 al 15 (1 para el valor más bajo y 15 para el valor más alto) (Toledo et al. 2016). Los datos presentados son el promedio de dos cosechas en años consecutivos ( $2^{\mathrm{a}}$ y $3^{\mathrm{a}}$ cosecha después de establecidos los cafetos).

\section{Análisis estadístico}

La producción de café cereza, la calidad física del grano y los rendimientos agroindustriales fueron evaluados mediante análisis de varianza, y se utilizó la prueba de Tukey para diferenciar las medias. En los rendimientos agroindustriales se llevaron a cabo pruebas ortogonales, planeadas con la prueba de $\mathrm{T}$ no pareada para comparar a las variedades Pluma Hidalgo 177 y Typica 947, con Blue Mountain (Di Rienzo et al. 2016). Por su calidad, otros tipos de Pluma Hidalgo y Typica son valorados en Oaxaca, y Blue Mountain en los mercados internacionales. La calidad sensorial de la bebida fue determinada por análisis de varianza no paramétrico, y para la comparación de medias se utilizó el método de Kruskal-Wallis (Di Rienzo et al. 2016).

\section{Resultados y Discusión}

\section{Producción de café cereza}

En las dos primeras cosechas, las variedades Oro Azteca, Caturra Rojo y Garnica F5 tuvieron mayor producción de fruta que las otras variedades (Cuadro 2). Los cafetos más productivos al inicio de su producción tienden a presentar buenos rendimientos entre los siguientes 5 y 10 años (Walyaro y Van der Vossen 1979). Así, al sumar la producción de seis cosechas bajo un manejo agronómico orgánico, las variedades con mayor producción fueron: Colombia Brote Café, Oro Azteca y Catuai Amarillo, todos de porte bajo (Cuadro 2). Las variedades Colombia Brote Café (López-García y Cruz-Castillo 2019) y Catuai Amarillo (López-García et al. 2016) también resultaron las más productivas en Veracruz, bajo un manejo agronómico convencional. En contraste, Oro Azteca con manejo convencional tuvo bajos rendimientos en varias regiones de Veracruz (López-García et al. 2016; López-García y Cruz-Castillo 2019). Sin embargo, en el presente estudio, Oro Azteca tuvo alta productividad con manejo orgánico.

Algunas variedades altamente productivas no son adecuadas para un manejo ecológico. Por otro lado, tampoco se debe descartar el uso de portainjertos para potenciar la mayor producción de C. arabica bajo manejo orgánico (López-García y Cruz-Castillo 2019). En la producción de café ecológico, los portainjertos podrían promover la producción (Myers et al. 2020). Catuai Amarillo es susceptible a la roya (Hemileia vastatrix Berk \& Br.) (https://varieties.worldcoffeeresearch.org/es/varieties/catuai; Escamilla-Prado 2016), pero las variedades Colombia con Brote Café y Oro Azteca, son derivadas del Híbrido de Timor $x$ Caturra y se han empleado para el control de la roya en Chiapas (Valencia et al. 2018). Además, con estas tres variedades de porte bajo, el número de plantas en la finca cafetalera puede ser incrementado.

En contraste, durante este periodo, la variedad con menor rendimiento $(\mathrm{P} \leq 0.05)$ fue Typica Tres Cruces. Typica comprende un grupo de plantas que usualmente tienen de baja a regular productividad. Sin embargo, en los cafetales de México existen Typicas sobresalientes que se adaptan bajo sombra y con un manejo de insumos bajos pueden durar más 
Cuadro 2. Producción de fruto en variedades de café durante seis cosechas en Oaxaca.

\begin{tabular}{|c|c|c|c|c|c|c|c|}
\hline \multirow[b]{2}{*}{ Variedad } & \multicolumn{6}{|c|}{ Cosechas (kg.planta-1) } & \multirow[b]{2}{*}{ Total } \\
\hline & 1 & 2 & 3 & 4 & 5 & 6 & \\
\hline Caturra Rojo & $0.1 \mathrm{ab}^{*}$ & $5.8 \mathrm{ab}$ & $7.5 \mathrm{bcde}$ & $3.826 a b c$ & $2.6 \mathrm{def}$ & $1.4 \mathrm{bcd}$ & $24.9 \mathrm{bcd}$ \\
\hline Catuaí Amarillo & $0.2 \mathrm{a}$ & $5.4 \mathrm{ab}$ & $8.1 \mathrm{abc}$ & $3.8 \mathrm{a}$ & 3.1 bcde & $1.4 \mathrm{bcd}$ & $27.0 \mathrm{ab}$ \\
\hline Caturra Amarillo & $0.2 \mathrm{a}$ & $5.3 \mathrm{abc}$ & 7.0 de & $3.7 \mathrm{abcd}$ & $2.5 \mathrm{ef}$ & $0.9 \mathrm{~cd}$ & 22.4 de \\
\hline Pacamara & $0.0 \mathrm{fg}$ & $2.1 \mathrm{efg}$ & $5.9 \mathrm{fgh}$ & 3.7 bcde & 2.5 ef & $0.8 \mathrm{~d}$ & $16.2 \mathrm{i}$ \\
\hline Garnica $\mathrm{F}_{5}$ & $0.1 \mathrm{abc}$ & $5.6 \mathrm{ab}$ & 8.0abcd & $3.8 \mathrm{ab}$ & $2.7 \mathrm{def}$ & $1.8 \mathrm{abcd}$ & $25.2 \mathrm{bcd}$ \\
\hline Garnica Iquimite & 0.0 de & $4.6 \mathrm{c}$ & 7.1 cde & $3.8 \mathrm{abc}$ & $2.7 \mathrm{def}$ & $1.6 \mathrm{abcd}$ & 24.0 cde \\
\hline Colombia (BV) & $0.1 \mathrm{~cd}$ & $4.5 \mathrm{c}$ & 7.1 cde & $3.8 \mathrm{abc}$ & $4.2 \mathrm{a}$ & $2.4 \mathrm{abc}$ & $26.5 \mathrm{bc}$ \\
\hline Colombia (BC) & $0.1 \mathrm{bcd}$ & $5.1 \mathrm{bc}$ & 8.9 a & $3.9 \mathrm{a}$ & $3.8 \mathrm{ab}$ & $2.9 \mathrm{a}$ & $30.2 \mathrm{a}$ \\
\hline Garnica (TCPB) & $0.0 \mathrm{def}$ & $5.1 \mathrm{bc}$ & $8.8 \mathrm{a}$ & 3.9 a & $2.2 \mathrm{f}$ & $1.9 \mathrm{abcd}$ & 24.8 cde \\
\hline Oro Azteca & $0.1 \mathrm{bcd}$ & $6.1 \mathrm{a}$ & $8.5 \mathrm{ab}$ & $3.9 \mathrm{a}$ & $2.6 \mathrm{def}$ & $2.2 \mathrm{abcd}$ & $29.0 \mathrm{a}$ \\
\hline Typica 947 & $0.0 \mathrm{fg}$ & 3.0 de & 6.7 ef & $3.7 \mathrm{abcd}$ & 2.5 ef & $2.7 \mathrm{ab}$ & $24.3 \mathrm{de}$ \\
\hline $\begin{array}{l}\text { Bourbón } \\
\text { Salvadoreño }\end{array}$ & $0.0 \mathrm{fg}$ & $1.5 \mathrm{fg}$ & 5.0 ghij & $3.6 \mathrm{def}$ & 2.9cdef & $2.2 \mathrm{abcd}$ & $19.9 \mathrm{gh}$ \\
\hline Batie & $0.0 \mathrm{fg}$ & $2.2 \mathrm{ef}$ & 5.1ghi & 3.6 cdef & $3.7 \mathrm{abc}$ & $2.7 \mathrm{ab}$ & 24.6 cde \\
\hline Blue Mountain & $0.0 \mathrm{fg}$ & $1.6 \mathrm{fg}$ & $3.6 j$ & $3.5 \mathrm{f}$ & $2.5 \mathrm{ef}$ & $2.3 \mathrm{abcd}$ & $17.5 \mathrm{i}$ \\
\hline Typica Xhantocarpa & $0.0 \mathrm{efg}$ & $1.8 \mathrm{fg}$ & $5.2 \mathrm{ghi}$ & 3.6 cdef & $2.6 \mathrm{def}$ & $1.6 \mathrm{abcd}$ & $20.5 \mathrm{fg}$ \\
\hline Pluma Hidalgo 177 & $0.0 \mathrm{efg}$ & $3.5 \mathrm{~d}$ & $4.8 \mathrm{hij}$ & 3.6 cdef & $3.5 \mathrm{abcd}$ & $3.2 \mathrm{a}$ & $24.0 \mathrm{ef}$ \\
\hline Dessie & $0.0 \mathrm{fg}$ & $2.0 \mathrm{efg}$ & $5.2 \mathrm{ghi}$ & 3.7 cdef & $4.2 \mathrm{a}$ & $2.8 \mathrm{ab}$ & 24.8 cde \\
\hline Garnica (TCPA) & $0.0 \mathrm{fg}$ & $2.0 \mathrm{efg}$ & $6.5 \mathrm{efg}$ & 3.7abcde & $2.9 \mathrm{bcdef}$ & $2.5 \mathrm{abc}$ & $21.0 \mathrm{fgh}$ \\
\hline Typica (TC) & $0.0 \mathrm{fg}$ & $1.2 \mathrm{~g}$ & $3.6 j$ & $3.5 \mathrm{f}$ & $2.1 \mathrm{f}$ & $1.1 \mathrm{bcd}$ & $13.4 \mathrm{j}$ \\
\hline Bourbón (TC) & $0.0 \mathrm{~g}$ & $1.5 \mathrm{fg}$ & $4.5 \mathrm{ij}$ & $3.6 \mathrm{ef}$ & 2.9bcdef & $2.3 \mathrm{abcd}$ & $18.0 \mathrm{hi}$ \\
\hline
\end{tabular}

*Medias con letras iguales de una misma columna no son estadísticamente diferentes (Tukey, 0.05).

de 40 años (Cruz y Torres 2015). La variedad Typica 947 tuvo una mediana producción de frutos (Cuadro 2). Un Typica muy apreciado por su calidad es el Kona que se cultiva en Hawai (Steiman et al. 2011), y otro cultivado en Oaxaca es la variedad Pluma Hidalgo, la cual es muy apreciada. También mostraron bajos rendimientos después de seis años las variedades de porte alto Pacamara y Blue Mountain, cuyo cultivo se ha extendido en Oaxaca (Cuadro 2).

\section{Calidad física del grano}

No hubo diferencias significativas $(\mathrm{P} \leq 0.05)$ entre las variedades en lo referente a la producción de frutos sin semilla, y el valor promedio a través de todas las variedades fue de 6.3 por ciento. La transformación de cereza a pergamino tampoco fue influenciada significativamente por la variedad (Cuadro 3). Sin embargo, en comparaciones ortogonales ( $\mathrm{P} \leq 0.05)$, las variedades Pluma Hidalgo 177 y Typica 947, con 216.4 kg y $219.1 \mathrm{~kg}$, respectivamente, presentaron buenos rendimientos cuando cada una fue comparada con Blue Mountain $(270.0 \mathrm{~kg})$. Espinosa-Solares et al. (2005) mostraron que la variedad Typica tuvo mayor eficiencia en conversiones de café cereza a pergamino, debido al mayor tamaño de sus frutos. Pluma Hidalgo 177 y Typica 947 estuvieron por debajo del estándar comercial de $245-250 \mathrm{~kg}$ de cereza para obtener 1 quintal de pergamino. El rendimiento de pergamino a oro (saco de $45.5 \mathrm{~kg}$ de café oro) de las 20 variedades 
Cuadro 3. Rendimientos agroindustriales en $\mathrm{kg}$ de cafetos en Oaxaca, promedio de dos años consecutivos.

\begin{tabular}{|c|c|c|}
\hline Variedad & Cereza a pergamino & Pergamino a oro \\
\hline Caturra Rojo & $239.1 a^{*}$ & $55.9 \mathrm{a}$ \\
\hline Catuaí Amarillo & $238.7 \mathrm{a}$ & $55.1 \mathrm{a}$ \\
\hline Caturra Amarillo & $250.0 \mathrm{a}$ & $55.9 \mathrm{a}$ \\
\hline Pacamara & $228.8 \mathrm{a}$ & $56.3 \mathrm{a}$ \\
\hline Garnica $F_{5}$ & 246.8 a & $56.1 \mathrm{a}$ \\
\hline Garnica Iquimite & $249.5 \mathrm{a}$ & $56.7 \mathrm{a}$ \\
\hline Colombia (BV) & $249.1 \mathrm{a}$ & $55.6 \mathrm{a}$ \\
\hline Colombia (BC) & $248.2 \mathrm{a}$ & $54.5 \mathrm{a}$ \\
\hline Garnica (TCPB) & $248.3 \mathrm{a}$ & $55.6 \mathrm{a}$ \\
\hline Oro Azteca & $246.1 \mathrm{a}$ & $55.6 \mathrm{a}$ \\
\hline Typica 947 & $219.1 \mathrm{a}$ & $56.6 \mathrm{a}$ \\
\hline Bourbón Salvadoreño & $239.8 \mathrm{a}$ & $56.8 \mathrm{a}$ \\
\hline Batie & $252.7 \mathrm{a}$ & $56.4 \mathrm{a}$ \\
\hline Blue Mountain & $270.0 \mathrm{a}$ & $56.2 \mathrm{a}$ \\
\hline Typica Xhantocarpa & $228.6 \mathrm{a}$ & $55.6 \mathrm{a}$ \\
\hline Pluma Hidalgo 177 & 216.4 a & $56.2 \mathrm{a}$ \\
\hline Dessie & $239.7 \mathrm{a}$ & $57.6 \mathrm{a}$ \\
\hline Garnica (TCPA) & $245.2 \mathrm{a}$ & $57.0 \mathrm{a}$ \\
\hline Typica (TC) & $232.9 \mathrm{a}$ & $56.7 \mathrm{a}$ \\
\hline Bourbón (TC) & $252.5 \mathrm{a}$ & $57.7 \mathrm{a}$ \\
\hline
\end{tabular}

*Medias con letras iguales de una misma columna no son estadísticamente diferentes (Tukey, 0.05).

no fue influenciado significativamente (Cuadro 3). Así, el manejo orgánico de los cafetales en los primeros años de producción no afectó $(\mathrm{P} \leq 0.05)$ estos dos parámetros industriales.

Las anormalidades en los granos fueron similares $(\mathrm{P} \leq 0.05)$ para todas las variedades (Cuadro 4). Resultados similares fueron determinados en Veracruz con estas variedades manejadas en forma convencional (López-García et al. 2016).

El tamaño comercial del grano de café en el mercado es determinado por los mayoristas (Díaz et al. 2009), aunque en Europa prefieren semilla grande. Las variedades Oro Azteca, Catuai Amarillo y Colombia Brote Café con alta productividad (Cuadro 2) también alcanzaron altos porcentajes de granos para la preparación europea (Cuadro 5). En cambio, las variedades con menor tamaño de grano y adecuadas para la preparación americana fueron Bourbón Salvadoreño y Bourbón Tres Cruces (Cuadro 5).

El tamaño del grano después de la cosecha afecta el proceso del tostado. Así, bajo un procesamiento similar, los granos más grandes tienden a presentar un tostado más ligero; en cambio, granos pequeños presentan un tostado más oscuro (Bosselmann et al. 2009), y esto influye en la apreciación sensorial del café en taza.

\section{Calidad sensorial de la bebida}

Se determinaron diferencias significativas en los atributos aroma, acidez, sabor y cuerpo. El aroma más intenso se obtuvo en las variedades Pacamara, Oro Azteca, Typica Xanthocarpa, Pacamara y Pluma Hidalgo 177 (Cuadro 6). La acidez fue elevada en variedades 
Cuadro 4. Porcentaje de anormalidades del grano de 20 variedades de café en Oaxaca, promediando dos años de producción.

\begin{tabular}{llllll}
\hline Variedad & Planchuela & Mancha & Caracol & Concha & Triangulo \\
\hline Caturra Rojo & $88.7 \mathrm{a}^{*}$ & $4.1 \mathrm{a}$ & $7.1 \mathrm{a}$ & $1.2 \mathrm{a}$ & $3.0 \mathrm{a}$ \\
Catuaí Amarillo & $85.0 \mathrm{a}$ & $3.9 \mathrm{a}$ & $8.2 \mathrm{a}$ & $0.8 \mathrm{a}$ & $5.9 \mathrm{a}$ \\
Caturra Amarillo & $87.0 \mathrm{a}$ & $2.6 \mathrm{a}$ & $5.3 \mathrm{a}$ & $1.6 \mathrm{a}$ & $6.0 \mathrm{a}$ \\
Pacamara & $85.2 \mathrm{a}$ & $4.6 \mathrm{a}$ & $8.3 \mathrm{a}$ & $1.0 \mathrm{a}$ & $5.5 \mathrm{a}$ \\
Garnica F & $85.3 \mathrm{a}$ & $2.3 \mathrm{a}$ & $8.0 \mathrm{a}$ & $3.6 \mathrm{a}$ & $4.6 \mathrm{a}$ \\
Garnica Iquimite & $87.1 \mathrm{a}$ & $3.1 \mathrm{a}$ & $5.3 \mathrm{a}$ & $1.1 \mathrm{a}$ & $6.5 \mathrm{a}$ \\
Colombia (BV) & $85.7 \mathrm{a}$ & $4.7 \mathrm{a}$ & $12.5 \mathrm{a}$ & $0.1 \mathrm{a}$ & $1.4 \mathrm{a}$ \\
Colombia (BC) & $86.0 \mathrm{a}$ & $3.8 \mathrm{a}$ & $7.7 \mathrm{a}$ & $2.2 \mathrm{a}$ & $4.3 \mathrm{a}$ \\
Garnica & $84.9 \mathrm{a}$ & $3.0 \mathrm{a}$ & $5.4 \mathrm{a}$ & $1.5 \mathrm{a}$ & $8.2 \mathrm{a}$ \\
Oro Azteca & $78.8 \mathrm{a}$ & $2.3 \mathrm{a}$ & $16.1 \mathrm{a}$ & $1.0 \mathrm{a}$ & $4.1 \mathrm{a}$ \\
Typica 947 & $86.2 \mathrm{a}$ & $3.7 \mathrm{a}$ & $6.5 \mathrm{a}$ & $0.2 \mathrm{a}$ & $5.5 \mathrm{a}$ \\
Bourbón Salvadoreño & $87.6 \mathrm{a}$ & $3.9 \mathrm{a}$ & $4.4 \mathrm{a}$ & $0.4 \mathrm{a}$ & $7.5 \mathrm{a}$ \\
Batie & $86.9 \mathrm{a}$ & $5.1 \mathrm{a}$ & $11.9 \mathrm{a}$ & $0.2 \mathrm{a}$ & $0.9 \mathrm{a}$ \\
Blue Mountain & $90.3 \mathrm{a}$ & $5.0 \mathrm{a}$ & $8.4 \mathrm{a}$ & $0.2 \mathrm{a}$ & $1.0 \mathrm{a}$ \\
Typica Xhantocarpa & $89.1 \mathrm{a}$ & $3.8 \mathrm{a}$ & $7.7 \mathrm{a}$ & $0.5 \mathrm{a}$ & $2.7 \mathrm{a}$ \\
Pluma Hidalgo 177 & $87.7 \mathrm{a}$ & $6.4 \mathrm{a}$ & $10.4 \mathrm{a}$ & $0.4 \mathrm{a}$ & $1.5 \mathrm{a}$ \\
Dessie & $81.3 \mathrm{a}$ & $8.6 \mathrm{a}$ & $17.0 \mathrm{a}$ & $0.6 \mathrm{a}$ & $1.0 \mathrm{a}$ \\
Garnica (TCPB) & $76.6 \mathrm{a}$ & $4.8 \mathrm{a}$ & $8.0 \mathrm{a}$ & $1.6 \mathrm{a}$ & $13.7 \mathrm{a}$ \\
Typica (TC) & $80.6 \mathrm{a}$ & $4.8 \mathrm{a}$ & $13.3 \mathrm{a}$ & $0.7 \mathrm{a}$ & $5.4 \mathrm{a}$ \\
Bourbón (TC) & $78.4 \mathrm{a}$ & $6.4 \mathrm{a}$ & $9.0 \mathrm{a}$ & $1.9 \mathrm{a}$ & $10.7 \mathrm{a}$ \\
\hline
\end{tabular}

*Medias con letras iguales de una misma columna no son estadísticamente diferentes (Tukey, 0.05).

como Pacamara, Colombia Brote Café, Colombia Brote Verde, Oro Azteca, Typica Xhantocarpa, Blue Mountain y Pluma Hidalgo 177, entre otras. En contraste, Garnica Tres Cruces Porte Bajo fue la variedad con menor aroma y acidez. El sabor también fue diferente $(\mathrm{P} \leq$ 0.05) para todas las variedades, y sobresalieron Oro Azteca y Catuai Amarillo. La composición bioquímica de los granos de café está influenciada por factores genéticos (Montagnon et al. 1998) y ambientales (Cheng et al. 2016); sin embargo, la calidad en taza de café producido por pequeños productores puede estar más relacionada con el microclima generado por el sistema de cultivo agroforestal utilizado (Bosselmann et al. 2009), y por la relación número de hojas: fruto por planta (Echeverría-Beirute et al. 2018). Así, los datos sobre calidad mostrados en otros estudios son muy variables y difíciles de comparar con nuestros resultados. En el presente estudio, se evaluó la calidad sensorial en taza durante dos cosechas consecutivas (al segundo y tercer año de su establecimiento), cuando los cafetos todavía no alcanzaban un potencial productivo permanente; así, el efecto de las variedades en plena producción sobre el tamaño del grano y sus propiedades bioquímicas es limitado. Por lo tanto, la calidad en taza de los granos de café debe ser evaluada en plantas con una madurez productiva.

\section{Conclusiones}

Las variedades de porte bajo Colombia Brote Café, Catuai Amarillo y Oro Azteca mostraron alta productividad durante sus primeros seis años de 
Cuadro 5. Tamaño del grano de café (\%) para preparaciones europea y americana, promediando dos años consecutivos.

\begin{tabular}{lll}
\hline \multirow{2}{*}{ Variedad } & \multicolumn{2}{c}{ Promedio $(\%)$} \\
\cline { 2 - 3 } & \multicolumn{2}{c}{ Europea } \\
\hline Caturra Rojo & $80.8 \mathrm{abc}^{*}$ & $19.1 \mathrm{bcd}$ \\
Catuai Amarillo & $82.2 \mathrm{abc}$ & $17.7 \mathrm{bcd}$ \\
Caturra Amarillo & $79.7 \mathrm{abc}$ & $20.2 \mathrm{bcd}$ \\
Pacamara & $91.7 \mathrm{a}$ & $8.2 \mathrm{e}$ \\
Garnica F5 & $75.4 \mathrm{abcd}$ & $24.6 \mathrm{abc}$ \\
Garnica Iquimite & $80.5 \mathrm{abc}$ & $19.4 \mathrm{bcd}$ \\
Colombia (BV) & $79.1 \mathrm{abc}$ & $20.8 \mathrm{bcd}$ \\
Colombia (BC) & $81.8 \mathrm{abc}$ & $18.2 \mathrm{bcd}$ \\
Garnica (TCPB) & $81.9 \mathrm{abc}$ & $18.0 \mathrm{bcd}$ \\
Oro Azteca & $83.5 \mathrm{ab}$ & $16.4 \mathrm{~cd}$ \\
Typica 947 & $75.4 \mathrm{abcd}$ & $24.5 \mathrm{abcd}$ \\
Bourbón Salvadoreño & $66.6 \mathrm{~cd}$ & $33.4 \mathrm{ab}$ \\
Batie & $84.4 \mathrm{ab}$ & $15.5 \mathrm{~cd}$ \\
Blue Mountain & $85.2 \mathrm{ab}$ & $14.7 \mathrm{~cd}$ \\
Typica Xhantocarpa & $77.7 \mathrm{abc}$ & $22.2 \mathrm{bcd}$ \\
Pluma Hidalgo 177 & $89.4 \mathrm{ab}$ & $10.5 \mathrm{~cd}$ \\
Dessie & $81.7 \mathrm{abc}$ & $18.2 \mathrm{bcd}$ \\
Garnica (TCPA) & $74.1 \mathrm{bcd}$ & $25.4 \mathrm{abc}$ \\
Typica (TC) & $74.0 \mathrm{bcd}$ & $25.9 \mathrm{abc}$ \\
Bourbón (TC) & $60.6 \mathrm{~d}$ & $39.3 \mathrm{a}$ \\
\hline
\end{tabular}

*Medias con letras iguales de una misma columna no son estadísticamente diferentes (Tukey, 0.05).

crecimiento y desarrollo, bajo un manejo agronómico orgánico. Tuvieron alrededor de $30 \mathrm{~kg} /$ planta. Estas tres variedades preliminarmente pueden ser recomendadas localmente en el Municipio de Valle Nacional en Oaxaca para ser producidas bajo un manejo orgánico. De estas tres variedades, Oro Azteca sobresale por presentar una calidad alta en taza. Las variedades de porte alto Typica 947 y Pluma Hidalgo 177 tuvieron un mediano rendimiento. Las variedades comúnmente usadas en Oaxaca como Typica y Bourbón fueron superadas en producción por variedades de porte bajo que pueden incrementar la densidad de plantas por hectárea. 
Cuadro 6. Catación en taza para variedades de café bajo producción orgánica en Oaxaca, México. Los valores son un promedio de dos cosechas en años consecutivos.

\begin{tabular}{lllll}
\hline Variedad & \multicolumn{1}{c}{ Aroma } & \multicolumn{1}{c}{ Acidez } & \multicolumn{1}{c}{ Sabor } & \multicolumn{1}{c}{ Cuerpo } \\
\hline Caturra Rojo & 11.1 ghij & $11.0 \mathrm{bcde}$ & $6.0 \mathrm{abc}$ & $9.4 \mathrm{abcd}$ \\
Catuai Amarillo & $10.8 \mathrm{hij}$ & $10.1 \mathrm{cdef}$ & $6.4 \mathrm{abc}$ & $8.5 \mathrm{de}$ \\
Caturra Amarillo & $10.1 \mathrm{jk}$ & $10.0 \mathrm{def}$ & $6.6 \mathrm{ab}$ & $8.7 \mathrm{bcde}$ \\
Pacamara & $13.2 \mathrm{abc}$ & $13.5 \mathrm{ab}$ & $5.8 \mathrm{bc}$ & $10.6 \mathrm{a}$ \\
Garnica F & $11.4 \mathrm{fghi}$ & $11.5 \mathrm{bcde}$ & $6.1 \mathrm{abc}$ & $9.4 \mathrm{abcd}$ \\
Garnica Iquimite & $10.3 \mathrm{ijk}$ & $9.1 \mathrm{ef}$ & $6.5 \mathrm{ab}$ & $8.5 \mathrm{cde}$ \\
Colombia (BV) & $12.3 \mathrm{bcdefg}$ & $12.0 \mathrm{abcd}$ & $5.6 \mathrm{bc}$ & $10.4 \mathrm{abc}$ \\
Colombia (BC) & $12.0 \mathrm{cdefgh}$ & $12.2 \mathrm{abcd}$ & $5.3 \mathrm{bc}$ & $10.2 \mathrm{abcd}$ \\
Garnica (TCPB) & $9.2 \mathrm{k}$ & $7.8 \mathrm{f}$ & $7.4 \mathrm{a}$ & $7.0 \mathrm{e}$ \\
Oro Azteca & $14.1 \mathrm{a}$ & $14.3 \mathrm{a}$ & $6.1 \mathrm{abc}$ & $11.0 \mathrm{a}$ \\
Typica 947 & $12.7 \mathrm{abcde}$ & $12.6 \mathrm{abcd}$ & $5.4 \mathrm{bc}$ & $9.7 \mathrm{abcd}$ \\
Bourbón Salvadoreño & $10.8 \mathrm{hij}$ & $11.1 \mathrm{bcde}$ & $6.1 \mathrm{abc}$ & $9.4 \mathrm{abcd}$ \\
Batie & $11.6 \mathrm{efgh}$ & $11.3 \mathrm{bcde}$ & $6.2 \mathrm{abc}$ & $9.3 \mathrm{abcd}$ \\
Blue Mountain & $12.3 \mathrm{bcdefg}$ & $13.1 \mathrm{ab}$ & $5.2 \mathrm{bc}$ & $10.2 \mathrm{abcd}$ \\
Typica Xhantocarpa & $13.6 \mathrm{ab}$ & $13.7 \mathrm{ab}$ & $6.1 \mathrm{abc}$ & $10.6 \mathrm{ab}$ \\
Pluma Hidalgo 177 & $13.1 \mathrm{abcd}$ & $13.4 \mathrm{ab}$ & $6.2 \mathrm{abc}$ & $10.5 \mathrm{abc}$ \\
Dessie & $12.5 \mathrm{bcdef}$ & $13.2 \mathrm{ab}$ & $5.5 \mathrm{bc}$ & $9.7 \mathrm{abcd}$ \\
Garnica (TCPA) & $12.7 \mathrm{abcde}$ & $13.3 \mathrm{ab}$ & $5.1 \mathrm{c}$ & $10.4 \mathrm{abcd}$ \\
Typica (TC) & $12.3 \mathrm{bcdefg}$ & $12.7 \mathrm{abc}$ & $5.8 \mathrm{abc}$ & $9.6 \mathrm{abcd}$ \\
Bourbón (TC) & $11.8 \mathrm{defgh}$ & $12.3 \mathrm{abcd}$ & $6.2 \mathrm{abc}$ & $9.5 \mathrm{abcd}$ \\
\hline
\end{tabular}

*Medias con letras iguales de una misma columna no son estadísticamente diferentes (Tukey, 0.05). 


\section{Literatura Citada}

Bosselmann AS, Dons K, Oberthur T, Olsen CS, Raebild A, Usma H. 2009. The influence of shade trees on coffee quality in small holder coffee agroforestry systems in Southern Colombia. Agriculture, Ecosystems \& Environment 129: 253-260. https://doi.org/10.1016/j. agee.2008.09.004

[CERTIMEX] Certificadora Mexicana de Productos y Procesos Ecológicos, S.C. [internet]. 2014. Normas para la producción, el procesamiento y la comercialización de productos ecológicos. [citado 2017 mar 12]. Disponible en: https://certimexsc.com/ docs/Normas\%20CERTIMEX\%20actualizadas\%20 10.2014\%20FINAL.pdf

Cheng B, Furtado A, Smyth HE, Henry RJ. 2016. Influence of genotype and environment on coffee quality. Trends in Food Science \& Technology 57: 20-30. https://doi. org/10.1016/j.tifs.2016.09.003

Cruz S, Torres C. 2015. El conocimiento campesino del agroecosistema cafetalero en la sierra sur de Oaxaca. Revista Mexicana de Ciencias Agrícolas 2: 147-154.

Di Rienzo JA, Casanoves F, Balzarini MG, González L, Tablada M, Robledo CW. 2016. InfoStat versión 2016. Grupo InfoStat/Universidad Nacional de Córdoba. Córdoba, Argentina.

Díaz R, Eakin H, Castellanos E, Jiménez G. 2009. Condiciones para la adaptación de los pequeños productores de café ante presiones económicas mediante procesos de "upgrading" en la cadena productiva. Revista Iberoamericana de Economía Ecológica 10: 61-72.

Echeverría-Beirute F, Murray SC, Klein P, Kerth C, Miller R, Bertrand B. 2018. Rust and thinning management effect on cup quality and plant performance for two cultivars of Coffea arabica L. Journal of Agricultural and Food Chemistry 66: 5281-5292. https://doi.org/10.1021/ acs.jafc.7b03180

Escamilla E, Ruiz O, Zamarripa A, González VA. 2015. Calidad en variedades de café orgánico en tres regiones de México. Revista de Geografía Agrícola 55: 45-55.

Escamilla-Prado E. 2016. Las variedades de café en México ante el desafío de la roya. Breves de Políticas Públicas 4: 1-10.

Espinosa-Solares T, Cruz-Castillo JG, Montesinos-López OA, Hernández-Montes A. 2005. Raw coffee processing yield affected more by cultivar than by harvest date. The
Journal of Agriculture of the University of Puerto Rico 89: 169-180. https://doi.org/10.46429/jaupr.v89i3-4.1038

Kasongo RK, Verdoodt A, Kanyankagote P, Baert G, Van Ranst E. 2011. Coffee waste as an alternative fertilizer with soil improving properties for sandy soils in humid tropical environments. Soil Use and Management 27: 94-102. https://doi.org/10.1111/j.1475-2743.2010.00315.x

Lazcano-Sánchez E, Trejo-Márquez MA, VargasMartínez MG, Pascual-Bustamante S. 2015. Contenido de fenoles, cafeina y capacidad antioxidante de granos de café verdes y tostados de diferentes Estados de México. Revista Iberoamericana de Tecnología Postcosecha 16: 293-298.

Lewis J, Runsten D. 2008. Is fair trade-organic coffee sustainable in the face of migration? evidence from a Oaxacan community. Globalizations 5: 275-290. https:// doi.org/10.1080/14747730802057738

López-García FJ, Cruz-Castillo JG. 2019. Yield of Coffea arabica grafted onto Coffea canephora in soils infested with nematodes in Mexico. Coffee Science 14: 308-314.

López-García FJ, Escamilla-Prado E, Zamarripa-Colmenero A, Cruz-Castillo JG. 2016. Producción y calidad en variedades de café (Coffea arabica L.) en Veracruz, México. Revista Fitotecnia Mexicana 39: 297-304.

Montagnon C, Guyot B, Cilas C, Leroy T. 1998. Genetic parameters of several biochemical compounds from green coffee, Coffea canephora. Plant Breeding 117: 576-578. https://doi.org/10.1111/j.1439-0523.1998.tb02211.x

Morales I. 2013. La vida en torno al café: marginación social de pequeños productores en San Pedro Cafetitlán, Oaxaca. México. Diálogos Revista Electrónica de Historia 14: 79-96.

Myers R, Kawabata A, Cho A, Nakamoto ST. 2020. Grafted coffee increases yield and survivability. HortTechnology 30: 428-432. https://doi.org/10.21273/ HORTTECH04550-20

Orozco FH, Cegarra J, Trujillo LM, Roig A. 1996. Vermicomposting of coffee pulp using the earthworm Eisenia fetida: Effects on $\mathrm{C}$ and $\mathrm{N}$ contents and the availability of nutrients. Biology and Fertility of Soils 22: 162-166. https://doi.org/10.1007/BF00384449

Partida-Sedas JG, Muñoz-Ferreiro MN, Vázquez-Odériz ML, Romero-Rodríguez MA, Pérez-Portilla E. 2019. Influence of the postharvest processing of the "Garnica" coffee variety on the sensory characteristics and overall acceptance of the beverage. Journal of Sensory Studies 34: e12502. https://doi.org/10.1111/joss.12502 
Rosas J, Escamilla E, Ruiz O. 2008. Relación de los nutrimentos del suelo con las características físicas y sensoriales del café orgánico. Terra Latinoamericana 26: 375-384.

Sánchez G, Olguín J, Mercado G. 1999. Accelerated coffee pulp composting. Biodegradation 10: 35-41. https://doi. org/10.1023/A:1008340303142

[SEMARNAT] Secretaria de Medio Ambiente y Recursos Naturales. [internet]. 2002. Norma Oficial Mexicana NOM-021-SEMARNAT-2000. Establece las especificaciones de fertilidad, salinidad y clasificación de suelos, estudio, muestreo y análisis. Mexico. [citado 2015 feb 27]. Disponible en: http://biblioteca.semarnat.gob.mx/ janium/Documentos/Ciga/libros2009/DO2280n.pdf.

[SIAP] Servicio de Información Agroalimentaria y Pesquera. [internet]. 2017. Cierre de la producción agrícola por estado 2015. [citado 2018 mar 20]. Disponible en: http:// infosiap.siap.gob.mx/aagricola_siap_gb/icultivo/.

Sosa L, Escamilla-Prado E, Díaz CS. 2004. Organic coffee. En: Wintgens JN, editor. Coffee: Growing, Processing, Sustainable Production. Weinheim, Wiley-VCH. P. 339-354.

Steiman S, Idol T, Bittenbender HC, Gautz L. 2011. Shade coffee in Hawai॰i-Exploring some aspects of quality, growth, yield, and nutrition. Scientia Horticulturae 128: 152-158. https://doi.org/10.1016/j.scienta.2011.01.011

Toledo PRAB, Pezza L, Pezza HR, Toci AT. 2016. Relationship between the different aspects related to coffee quality and their volatile compounds. Comprehensive Reviews in Food Science and Food Safety 15: 705-719. https://doi. org/10.1111/1541-4337.12205

Valencia J, Pinzón M, Gutiérrez R. 2015. Caracterizacion fisicoquímica y sensorial de tazas de café producidas en el Departamento del Quindío. Alimentos Hoy 23: 150-156.

Valencia V, García-Barrios L, Sterling EJ, West P, MezaJiménez A, Shahid N. 2018. Smallholder response to environmental change: Impacts of coffee leaf rust in a forest frontier in Mexico. Land Use Policy 79: 463-474. https://doi.org/10.1016/j.landusepol.2018.08.020

Valkila J. 2009. Fair Trade organic coffee production in Nicaragua-Sustainable development or a poverty trap? Ecological Economics 68: 3018-3025. https://doi. org/10.1016/j.ecolecon.2009.07.002

Van der Vossen HAM. 2005. A critical analysis of the agronomic and economic sustainability of organic coffee production. Experimental Agriculture 41: 449-473. https://doi.org/10.1017/S0014479705002863
Van der Vossen H, Bertrand B, Charrier A. 2015. Next generation variety development for sustainable production of arabica coffee (Coffea arabica L.): A review. Euphytica 204: 243-256. https://doi.org/10.1007/s10681-015-1398-z

Walyaro DJ, Van der Vossen HAM. 1979. Early determination of yield potential in arabica coffe by applying index selection. Euphytica 28: 565-472. https://doi. org/10.1007/BF00056606 